\title{
A novel silicon membrane-based biosensing platform using distributive sensing strategy and artificial neural networks for feature analysis
}

\author{
Zhangming $\mathrm{Wu}^{\mathrm{a}}$, Khujesta Choudhury ${ }^{\mathrm{b}}$, Helen R. Griffiths ${ }^{\mathrm{b}}$, Jinwu $\mathrm{Xu}^{\mathrm{c}}$, Xianghong Ma ${ }^{\mathrm{a}, *}$ \\ ${ }^{a}$ School of Engineering and Applied Science, Aston University, Birmingham B4 7ET, UK, \\ ${ }^{b}$ School of Life and Health Sciences, Aston University, Birmingham B4 7ET, UK, \\ ${ }^{c}$ Department of Mechanical Engineering, University of Science and Technology Beijing 100083 Beijing, China
}

\begin{abstract}
A novel biosensing system based on a micromachined rectangular silicon membrane is proposed and investigated in this paper. Distributive sensing scheme is designed to monitor the dynamics of the sensing structure.An artificial neural network is used to process the measured data and to identify cell presence and density. Without specifying any particular bio-application, the investigation is mainly concentrated on the performance testing of this kind of biosensor as a general biosensing platform. The biosensing experiments on the microfabricated membranes involve seeding different cell densities onto the sensing surface of membrane, and measuring the corresponding dynamics information of each tested silicon membrane in the form of a series of frequency response functions (FRFs). All of those experiments are carried out in a cell culture medium to simulate a practical working environment. The EA.hy 926 endothelial cell lines are chosen in this paper for the bio-experiments. The EA.hy 926 endothelial cell lines represent a particular class of biological particles that have unregular shapes, non-uniform density and uncertain growth behaviour, which are difficult to monitor using the traditional biosensors. The final predicted results reveal that the methodology of a neural-network based algorithm to perform the feature identification of cells from distributive sensory measurement, has great potential in biosensing applications.
\end{abstract}

Keywords: Biosensors, Microscale membrane, Distributive sensing, Neural network, Endothelial cell lines

\section{Introduction}

Research into the use of biosensors for the detection of various biological particles/molecules have received extensive interest in recent decades, due to the rapid progress of micro/nano technologies. A biosensor usually consists of a bioreceptor and a sensing transducer, in which the bioreceptor is the interface that the biosensor interacts with the biological environment and the transducer is used to convert the physical/chemical information of the biological particles into a measurable signal[1]. Microcantilevers are the most widely used transducer in mechanical-type biosensors, due to their ultra-small size, high sensitivity and label-free biological application by surface functionalization[2]. This kind of biosensor can be fabricated in arrays of microcantilevers[3] and can be integrated into a CMOSbased microsystem. In general, microcantilever can

\footnotetext{
Email address: x.ma@aston.ac.uk (Xianghong Ma)
}

work under two different modes: static mode and dynamic mode[4,5]. In a static mode, surface stresses are accumulated after binding biological particles to the microcantilever surface and it will inevitably increase the static deformation of microcantilever. The dynamic mode use a microbalance approach, which detects surface-attached mass using resonant frequency shift. However microcantilever based biosensors suffer from low sensitivity in liquid environment and fragility in practical operation[6].

Micromachined membranes (plate/diaphragm) have gradually become a promising mass sensing structure to replace the microcantilever in recent years. Compared with microcantilevers, micro-membranes potentially have larger sensing area, higher sensitivity in liquid and less fragility. Moreover, it has the same advantages as the microcantilever in the application of mass sensing. Some researchers have made attempts to apply micro-membranes in biological detections. For examples, Carlen et al [7] designed a micromachined surface stress sensor based on a thin suspended crystalline

September 1, 2010 
silicon circular plate to detect the bending behaviour caused by vapor phase chemisorption of the alkanethiol monolayers. $\mathrm{Xu}$ et al [6] developed a piezoelectric membrane-based biosensor array for immunoassay applications.

Distributive sensing techniques have been widely used to monitor and reconstruct the static deformation or dynamic responses of conventional structures, in the field of vibration control, damage detection and biomedical analysis etc. One famous instance is a beamlike or plate-like smart sensing surface with few distributive tactile sensors. The sensors are placed at selected locations and used to collect the data of surface deformation. Any change upon the sensing surface can result in a corresponding change of measurements in each sensor. The features or the properties of contacted object are related to the sensory data. Advanced nonlinear feature analysis methods, for example artificial neural networks, can be applied to infer the properties of the contacted subject. These kinds of tactile sensing surfaces have been successfully applied to determine a description of force loading[8], localize a contacting subject[9] or even human gait analysis[10]. Apparently the design of integrated microsystems for biosensing can also borrow the concept of the smart sensing surface, in which multi-dimensional signals rather than single output from the sensing surface can be collected and used for post-processing. Therefore it has good potential to extract more information such as distribution or pattern of biological targets, rather than just the mass variations.

Frequency response function (FRF) is one of the most useful ways to represent the dynamics of a rectangular membrane. Cell adhesion on a membrane surface results in the change of its mass, stiffness and damping, all of which can be reflected in a FRF. By comparing the FRFs of a sensing membrane with and without cell attachment, we can identify the features of adhesive cells. Many researchers have successfully employed neural networks on the measued FRF data for structural health monitoring and damage detection[11, 12, 13, 14]. This paper presents the successful cell monitoring application. Before applying FRF data into training a neural network, the size of FRF data has to be reduced. Many methods exist to perform the data dimension reduction, such as sub-dataset[11], modal analysis[15] and principal component analysis[12]. In this paper, KarhunenLoeve decomposition is used for FRF data dimensionality reduction.

The purpose of this paper is to apply a micromachined rectangular membrane as biosensing platform using the distributive sensing method. This novel ap- proach is first in the field of biosensing, which paves the way for developing more advanced biosensors with high accuracy and multiple functions. The paper is organised as follows. Section 2 presents the fabrication methods of biosensing platform including the distributive piezoresistive sensors and PZT actuators for self-actuation and self-sensing. Section 3 presents the process of biological testing. Experimental tests of biosensing micro-membranes are used for detection of EA.hy 926 endothelial cell lines in a natural liquid environment. EA.hy 926 is a well-established human endothelial-like immortalised cell line that exhibits adherence and migratory characteristics, resulting in nonuniform shapes in culture. Dynamic responses of the micro-membrane at a few specific points are measured and recorded. Such information forms a set of distributive sensory data. In analysing the sensory data, first a shift of resonance frequency at each measured mode is used to perform a preliminary estimation of the cell density. It is found that frequency based indices alone is unable to accurately reflect the attached cell distribution on the sensing surface. Finally, in Section 4, a Back-Propagation (BP) neural network with one hidden layer is trained to recognize the cell distribution from the distributive sensory data of a series of repeated bioexperiments. It shows precise prediction on cell density by using this neural network model.

\section{Fabrication of membrane biosensing devices}

The silicon membranes were fabricated using the standard micromachining techniques from silicon on insulator (SOI) wafers. The membrane was created by inductively coupled plasma (ICP) using the Deep Reactive Ion etching (DRIE) process from the back side of SOI wafer, stopping at the buried oxide layer. Boundary conditions of the membrane were also defined by DRIE from the top side of the wafer, using the buried oxide as stop layer. The buried oxide layer was finally removed to form the boundary holes. Figure 1 illustrates an approximate $200 \mu m$ square membrane of cantilever structure. Three different boundary conditions of the micro-membranes were fabricated and tested: two opposite edges clamped and the other two edges free (C-F-C-F), cantilever (C-F-F-F) and all edges clamped (C-C-C-C). All of the membranes are designed to be square and with lengths of $100 \mu \mathrm{m}, 200 \mu \mathrm{m}$ or $300 \mu \mathrm{m}$.

Figure 2 demonstrates an integrated microsystem based on a square sensing membrane, which was manufactured with distributive piezoresistive sensors and PZT actuators. Such a microsystem enables the device to be capable of self-sensing and self-excitation. This 
microsystem can be embedded into an electronic circuit to build a lab-on-chip system.

For the fabrication of distributive piezoresistive sensors, a $500 \mathrm{~nm}$-thick poly-silicon layer was deposited onto the oxidised device layer of a SOI wafer by low pressure chemical vapour deposition (PCVD). This layer was then doped by ion beam implantation using a $50 \mathrm{Kev}$ Boron source giving a doping density of $1 \mathrm{e} 15$ to enhance the piezoresistive deflection sensitivity. The sensor shapes were formed by photo-lithography and subsequent reactive ion etching (RIE).

In the PZT film fabrication, a sandwiched structure of a $100 \mathrm{~nm}$-thick Pt/Ti bottom electrode, a $1 \mu \mathrm{m}$ PZT film and a $100 \mathrm{~nm}$-thick Pt top electrode was deposited on the SOI. The top and bottom electrodes were deposited by evaporation using e-beam evaporator systems, the deposited PZT was deposited as a spin on sol-gel which is then annealed to produce the required PZT film. The top and bottom electrodes are patterned and etched by ion beam milling. The redundant PZT material was wet etched.

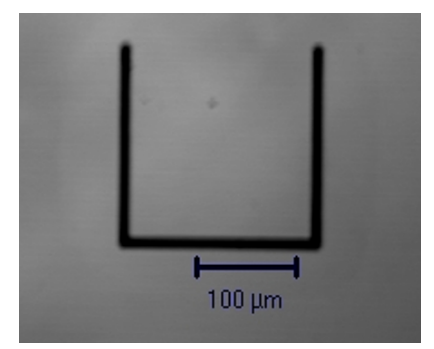

Figure 1: Laser scanning image of a $200 \mu \mathrm{m}$ square pure membrane of cantilever structure

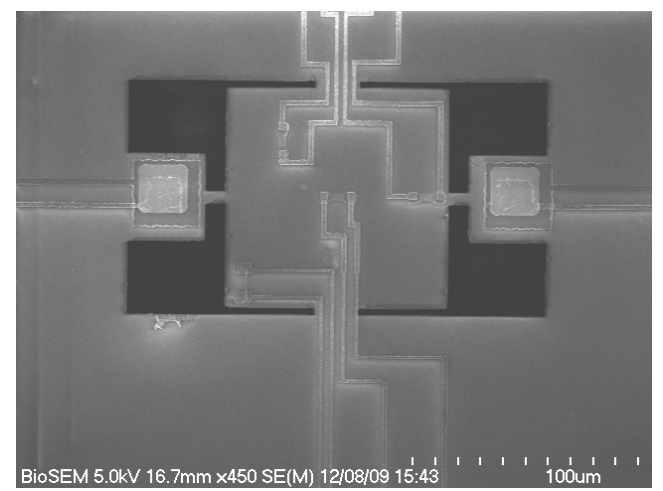

Figure 2: SEM image of an integrated microsystem using a $100 \mu m$ square membrane and attaching with distributive piezoresistive sensors and PZT actuators

\section{Biological experiments}

\subsection{The process of bio-experiments}

The human hybrid EA.hy 926 cell used in this paper is derived from the fusion of the human umbilical vein endothelial cells with A549/8 human lung carcinoma cell line. EA.hy 926 is a permanent human endothelial cell line that expresses highly differentiated functions characteristic of human vascular endothelium. Human EA.hy 926 endothelial cell lines are maintained in 30ml Dulbecco's Modified Eagle's Medium (DMEM), supplemented with $10 \%$ FBS, streptomycin $100 \mu \mathrm{g} / \mathrm{ml}$ and penicillin $100 \mathrm{U} / \mathrm{ml}$, and $10 \mathrm{ml}$ HAT $(100 \mu \mathrm{M}$ hypoxanthine, $0.4 \mu M$ aminopterin, $16 \mu M$ thymidine). Cells were cultured in an incubator at $37^{\circ} \mathrm{C}$ with an atmosphere of $5 \% \mathrm{CO}_{2}$ and $95 \%$ air. Cells were grown in a $75 \mathrm{~cm}^{2}$ flask and passaged when reaching $\sim 90 \%$ confluence. Once cells roughly reached $90 \%$ confluence the media was removed and the cells washed with $5 \mathrm{ml}$ phosphate buffered saline (PBS). The process of passage of EA.hy 926 cells is that briefly cell culture media was removed from the cells and cells were then washed with $10 \mathrm{ml}$ sterile PBS until the media appears without color. EA.hy 926 cells were then detached by the addition of $2.5 \mathrm{ml}$ trypsin with a 3 minute standard incubation. Cell clusters were also dispersed for uniform distribution by repeated pipetting with $5 \mathrm{ml}$ new DMEM media.

Figure 3 shows a LSM image that the endothelial cells coated on the surface of a micro-membrane. It can be seen that those endothelial cells were tightly adhered to the silicon surface showing a typical spreading pattern.

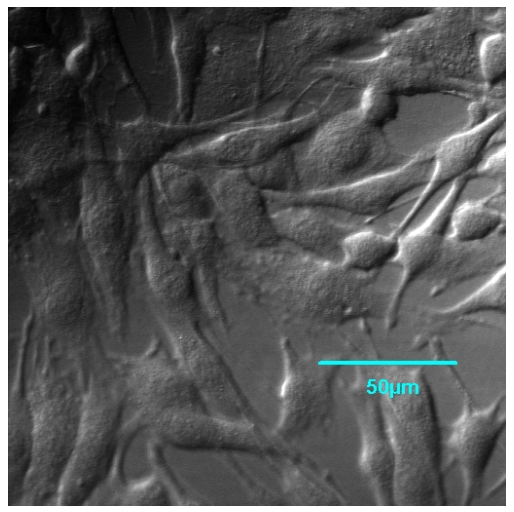

Figure 3: Laser scanning image of endothelial cells coating on the surface of a micro-membrane

The seeding of biological experiment is separated into two phases: seeding a certain amount of cells on the 
membrane (Figure 4-a,b) and measure the corresponding dynamics of this membrane. The dynamic testing device is illustrated in Figure 4-c. Identical micromembranes were repeatedly used several times for obtaining a batch of experimental results with different densities of cells. Each experiment was performed according to the following work flow:

1. Initially, silicon micromembranes were cleaned and sterilised using washes (ethanol and acetone mixture), autoclaving and UV light irradiation.

2. Before seeding cells on the micro-membranes, the cell density of suspension during the process of passage was established. The numbers of viable cells were estimated by taking $20 \mu \mathrm{l}$ of the cell suspension and mixing it with a $20 \mu l$ trypan blue. Cells count was then performed from this new mixture by using improved Neubauer haemocytometer. Once the cell density was established, a $5 \mathrm{ml}$ cell suspension of EA.hy 926 cells of known density is made up using the media. By controling the incubation time, various cell density and distribution on the membrane surface can then be achieved.

3. Cell distribution on the membrane sensing surface is recorded using a LSM (laser scan microscopy) image. The density or distribution of cells can be quantitated based on this LSM image.

4. The dynamics of membranes with adherent cells are measured. The FRF data for each specific micro-membrane with cells and without cells are compared to infer the information of cells, which is recorded in the LSM scanned images.

5. Finally, the cells are removed from the surface of micro-membranes following the same procedure as the first step. The re-sterilised micro-membrane can be used for the next experiment.

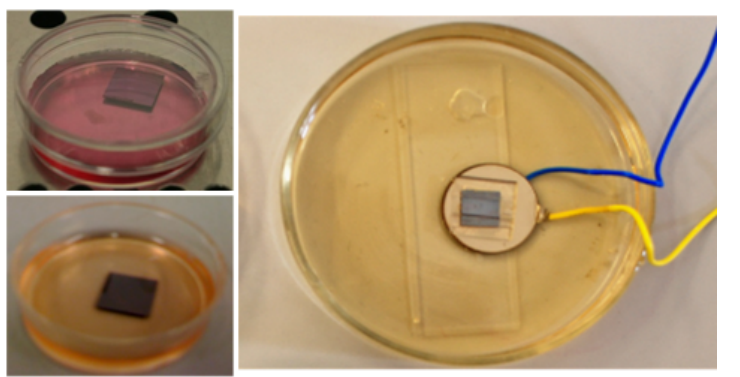

Figure 4: Endothelial cells coating on the surface of a micromembrane: (a)The left upper image shows a silicon die (membrane) inside in a petri dish, (b)The left bottom image shows the same after a period of incubation, (c)The right image is the dynamic testing device.

\subsection{Experimental results}

Figures 5, 6 and 7 illustrate the frequency response functions (FRFs) of three different types of micromembranes under three different cell densities. The most dominant change of the dynamics of membrane induced by cell-loading is the shift of resonance frequencies. As the first mode shapes remain almost constant[16], and the amplitudes of each FRF were selfnormalized with respect to the amplitude of first resonant mode. Relative amplitudes of resonant modes are found to be significantly varied after the cell loading. It means that additional mass loading of attached cells on the surface of membrane also results in the distortion of vibration shapes. The mass or quantity of target cells can be estimated through the detection of the shift of resonance frequencies. Eq. 1 demonstrates the relationship between mass change and frequency shift of a dynamic system, under the assumption that the stiffness remains constant. This approach has been widely used in the microcantilever based biosensors.

$$
f=\frac{1}{2 \pi} \sqrt{\frac{k}{m}}, \quad \frac{\Delta m}{m}=\frac{k}{4 \pi^{2}}\left(\frac{1}{f_{1}^{2}}-\frac{1}{f^{2}}\right) \approx 2 \frac{\Delta f}{f}
$$

Comparing the changes of FRFs presented in Figures 5,6 and 7, it is concluded that different types (dimension and boundary conditions) of the rectangular silicon micro-membranes reflect very different biosensing performance. It implies that the first type membrane (a 100 $\mu m$ square C-F-F-F) has highest sensitivity among the three membranes, in terms of resonance frequency shift. It is also noted that nonlinearity occurs on the dynamics of fluid-loaded micro-membranes. In fact most experimental results of FRFs micro-membranes involved cell attachment have suffered with nonlinearity to a certain degree. In general, the experimental results shown in Figures 5, 6 and 7 demonstrate the great potential ability of micro-membrane in biosensing, even when they are immersed in a high-damping liquid environment.

\subsection{Preliminary analysis}

Two resonant frequency based indices (Eq. 2) are utilized to perform a preliminary analysis on the experimental results in this paper. $F D R_{n}$ (Frequency Difference Ratio) is evaluated as the normalized resonant frequency difference between the cell-loaded and cell-free membrane at each measured resonance mode. $A F D R$ is the average of all measured $F D R_{n}$.

$$
F D R_{n}=\frac{\Delta f_{n}}{f_{n}}, \quad A F D R=\frac{1}{N} \sum_{n}^{N} F D R_{n}
$$



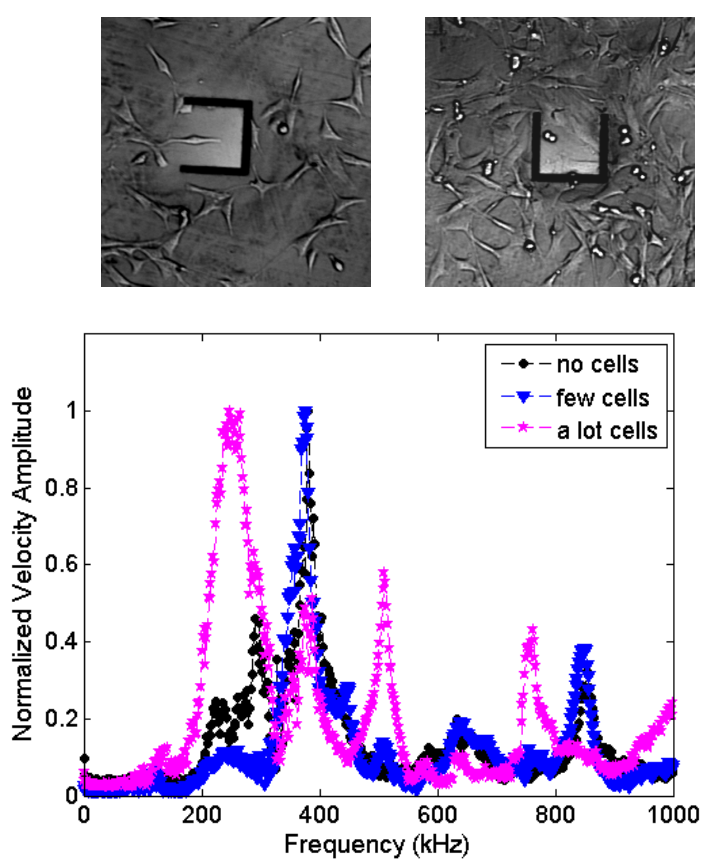

Figure 5: Above: endothelial cells coating on the surface of a 100 $\mu m$ square $C-F-F-F$ micro-membrane. Below: normalised velocity amplitude according to cell density.
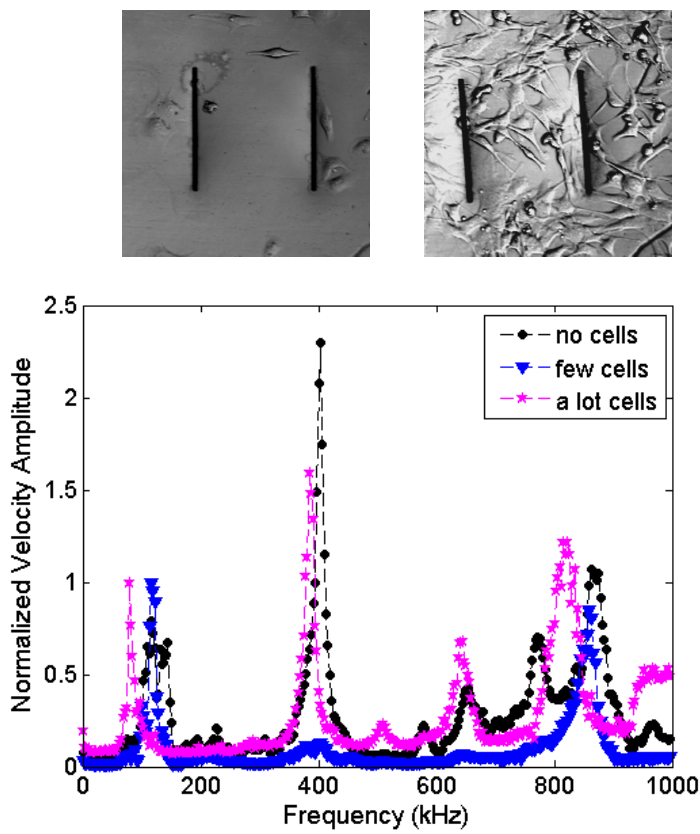

Figure 6: Above: endothelial cells coating on the surface of a 200 $\mu m$ square $C-F-C-F$ micro-membrane. Below: normalised velocity amplitude according to cell density.
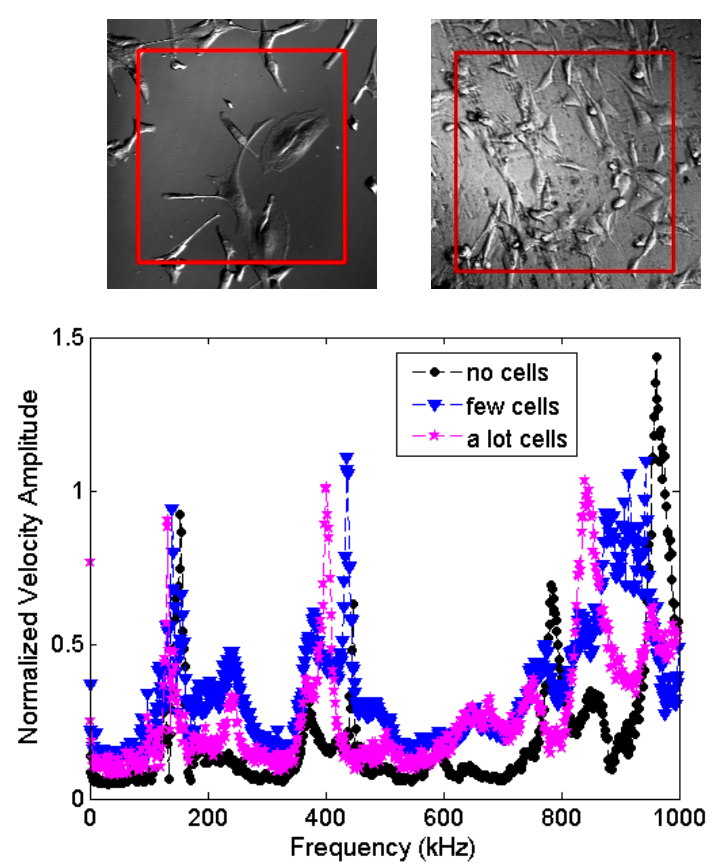

Figure 7: Above: endothelial cells coating on the surface of a 300 $\mu m$ square $C-C-C-C$ micro-membrane. Below: normalised velocity amplitude according to cell density.

The indices of $F D R_{n}$ and $A F D R$ evaluation were performed on three batches of bio-experimental results using three different micro-membranes, which are all approximate $200 \mu \mathrm{m}$ square C-F-C-F membranes. The three micro-membranes are labeled as No.I, No.II and No.III respectively. In each batch of the experiment, an identical membrane was repeatedly used four times and the cell culture density was gradually increased from to $25 \times 10^{3} / \mu l$ to $200 \times 10^{3} / \mu l$. Figures 8,9 and 10 illustrate the trends of the $F D R_{n}$ with increasing the amount of cells of each tested micro-membrane. Figure 11 compares the $A F D R$ index of these three micro-membranes in each batch of experiment.

First of all, some trends of the index $F D R_{n}$ at one or two modes are not coherent with the increase of cell quantity. This phenomenon is quite different with the bio-experimental results of microcantilever, where the $F D R_{0}$ of its fundamental mode always has a linearly relationship with cells number[17, 18]. The potential reasons of this phenomenon are: (a)Micro-membranes usually have much larger sensing area and carry many more cells than microcantilever in the bio-experiments. Apart from mass change, the accumulation of cells may also result in change of structural stiffness. In such cases, the linear relationship of FDR will be violated. (b)The bio-experiments presented in this work for 


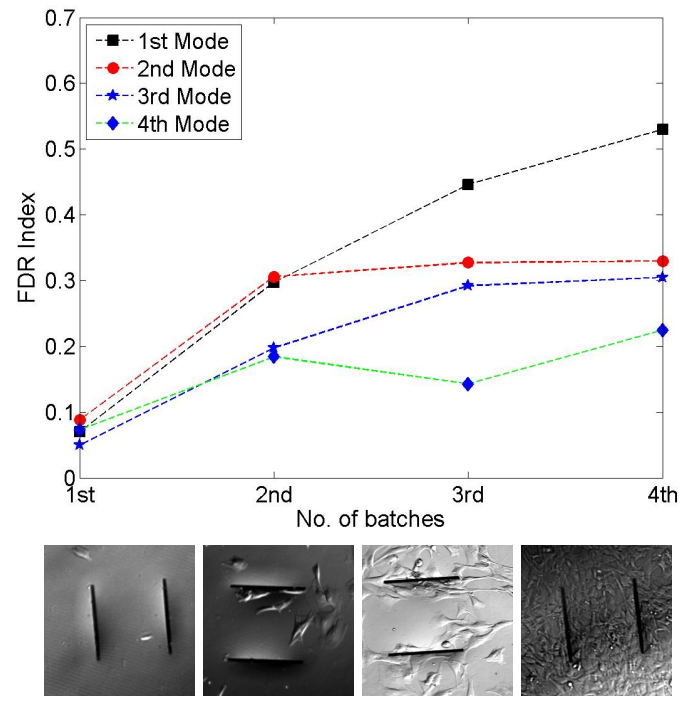

Figure 8: Above: FDR trends of No.I micro-membrane in four independent biological experiments. Below: typical cell growth observed at the four time intervals selected.

micro-membranes are maintained in a relevant environment, for example the dynamics of microplates are measured in cell culture media. (c)Nonlinearity of the dynamics of submerged micro-membranes with randomly distributed cells exists in most experimental measurements.

On the other hand, index $A F D R$ is capable of giving an approximate prediction of the amount of cells. The sensitivity of $A F D R$ on these three micro-membranes is quite different. The values of $A F D R$ for No. I and No. II membranes are very close, but that of No. III is much lower. This is due to the fact that No. I and No.II membranes were taken from the same wafer, while No.III is from the other one. It reveals that using the index $A F D R$ for the micro-membrane as a biosensing platform is not a robust method. Calibration on such a biosensing device is probably required before the estimation on cell density.

Considering the submerged sensing membrane as a general oscillation structure, resonant frequency can be approximately determined only by its stiffness and mass, the first equation in 1 . If one assumes the system stiffness is a constant, the mass change ratio is proportional with frequency change ratio as shown in second equation of 1 . It is therefore believed that indices $F D R_{n}$ and $A F D R$ are able to roughly reflect the cell density. However in realistic situations cells attachment would also affect the stiffness of sensing micromembrane more or less, especially the endothelial cells.
It leads to more complication and $F D R_{n}$ and $A F D R$ more difficult to accurately indicate the cells density.

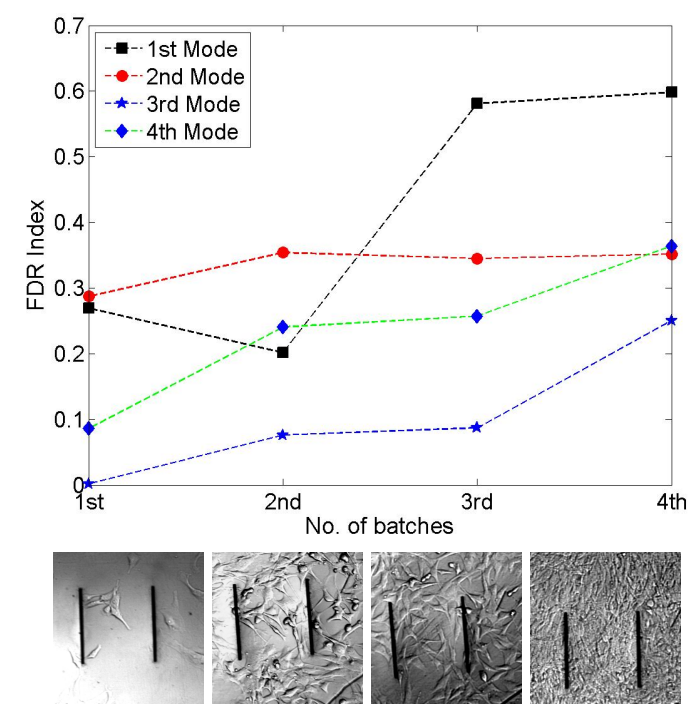

Figure 9: Above: FDR trends of No.II micro-membrane in four independent biological experiments. Below: typical cell growth observed at the four time intervals selected.

\section{Neural network method}

On the whole, resonant frequency based indices either $F D R_{n}$ or $A F D R$ are only able to predict the cell density with very limited accuracy. It is mainly due to the complication and nonlinearities of micro-membrane sensing system. Other algorithms are desired to perform more accurate and reliable identification on cell distribution from the measured dynamics data. In this section, a simple attempt that using an artificial neural network technique to build the relationship between the sensory data and cell distribution is carried out.

\subsection{Quantitation of cell density}

In the above experimental results, LSM images were used to intuitively presented the cell population in the micro-membrane sensing domain. However a quantitative index is also necessary to indicate the amount of cells for a more precise analysis. This is especially true for endothelial cells, the number of which are very hard to count. A simple image processing procedure was carried out on each LSM image to convert it into a binary image using the MATLAB Image Processing Toolbox. Initially the LSM image is loaded and a most clear layer is selected for the following processes, as 


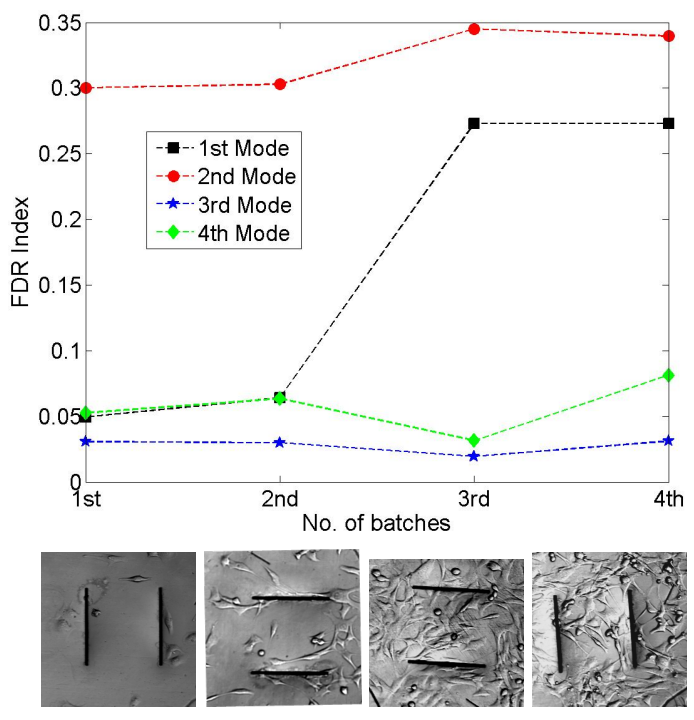

Figure 10: Above: FDR trends of No.III micro-membrane in four independent biological experiments. Below: typical cell growth observed at the four time intervals selected.

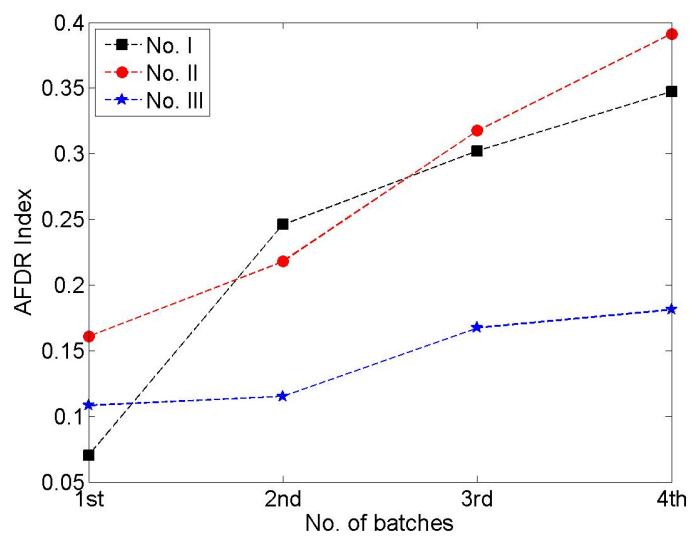

Figure 11: AFDR trends of three different micro-membranes in each batch of bio-experiments the LSM image taken under the reflection mode usually contains three layers. Then the background image of this LSM image is created by the morphological opening technique. Afterwards the background image is subtracted from the original image and the image contrast is enhanced, this is in order to highlight the area of cells occupied. Finally the corresponding binary image is created, in which the background is black and the parts of implanted cells are white. Therefore the cells population on the sensing domain can be approximately evaluated by the white area ratio in this binary image. This ratio is called cell density ratio (CDR) in this paper. Figure 12 demonstrates the results of this evaluation processes on four different LSM images, which are obtained in a same batch of bio-experiments. It can be seen that the white region of each binary image roughly indicates the shapes of endothelial cells distribution, although some local errors exist in the binary images. The evaluated ratios of white region are also listed in the bottom of Figure 12.

However, these evaluated CDRs are not suitable to be used directly in the analysis due to the following points: (1)Apart from each cell height above the growth surface, the endothelial cells also generate a thin film over all of the culture surface. Each evaluated CDR is raised up $10 \% \sim 15 \%$ to consider this thin film loading effect, for distinguishing from the case of no cells loading; (2) For the case that cells covered nearly the whole sensing domain, i.e. the 4th image in Figure 12, the predicted value of CDR is usually much lower than the actual situation. Therefore the predicted value needs to be increased. The modified CDRs for each experimental sample are then used as the target values in neural network applications.

\subsection{FRF Data Normalization and Order-Reduction}

Although all of experimental settings are the same in each time of dynamic experiment, the amplitudes of every FRF measurements are varied with experimental environment and external disturbances. Consequently it is better to normalize the measured FRFs and scale them into a same level for comparison and analysis. On the other hand, there are multiple FRF datasets in each dynamical measurement and each FRF dataset contains a very large number of frequency spectral lines. In this work, frequency spectral lines are set to be 6400 for each FRF and 4 sensory FRFs were recorded for each test. Obviously such FRF datasets are too large to directly apply into the neural network. Therefore the dimension of each FRF has to be reduced before the application of neural network. 

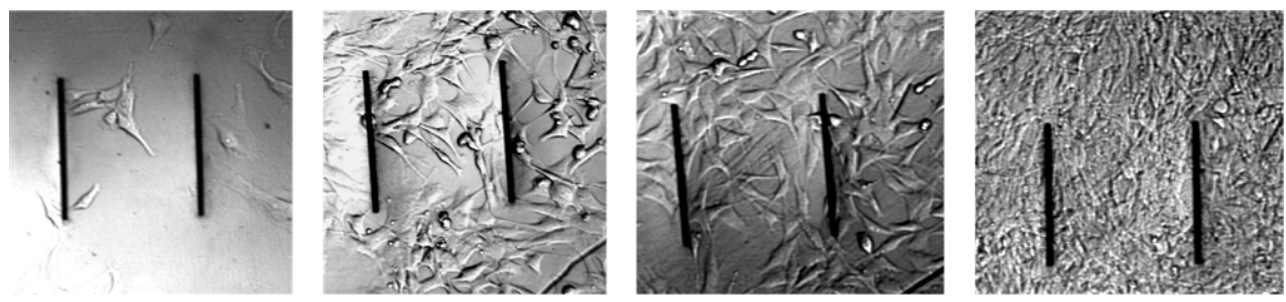

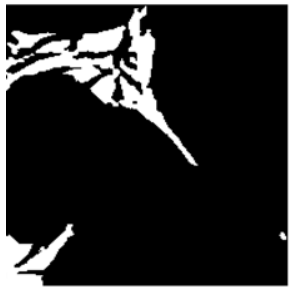

$10.46 \%$

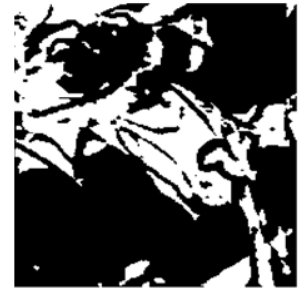

$30.07 \%$

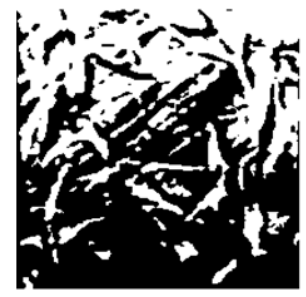

$39.69 \%$

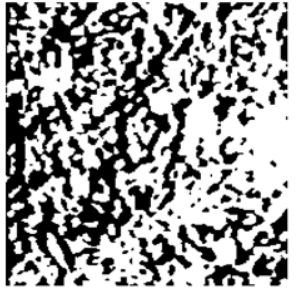

$58.02 \%$

Figure 12: Quantization of cells population based on a simple image process technique

For the FRF normalization, each spectrum is normalized with respect to the amplitude of its own first resonant mode. The reason for choosing the first resonant mode as the reference is based on the theoretical analysis results in [16], which prove that the mass loading has the slightest effects on the first resonant mode of a rectangular membrane.

For the dimensionality reduction, Karhunen-Loeve (K-L) decomposition method is then used to extract the principal components on a multiple-FRFs dataset. The Karhunen-Loeve (K-L) decomposition is a useful method to create low dimensional, reduced-order models of dynamical systems[19]. Assuming there are $M$ of FRFs with $N$ frequency in each of dynamics measurement of membrane, then this dataset forms a $M \times N$ matrix $[H(\omega)]_{M \times N}$. The process of principal component extraction of the matrix $[H(\omega)]$ using Karhunen-Loeve (K-L) method has the following steps:

1. Firstly, a correlation matrix $[C]_{M \times M}$ is created based on the FRF matrix $[H(\omega)]_{M \times N}$.

$$
[C]_{M \times M}=[H(\omega)]_{M \times N}[H(\omega)]_{N \times M}^{T}
$$

2. The principal components are then obtained from calculating the eigenvalues and corresponding eigenvectors of matrix $[C]$.

$$
[C][X]=\lambda[X]
$$

3. Finally, the $M$ extracted eigenvalues are examined. The eigenvectors associated with these largest eigenvalues are then considered to be the principal components and be able to represent the most significant information of the original FRF dataset.

\subsection{Dataset creation}

The dynamics (FRF) of 4 different used membranes without any cells loading are also provided in the dataset as references. Two additional samples are also provided for the purpose of validation. Consequently there are 18 different samples in total are created for training and validation of the neural network. The eigenvectors related to the largest eigenvalue of FRF dataset of each sample are extracted as the neural network input and the CDRs of every samples are calculated as the neural network targets.

\subsection{Network design and training}

The widely used back-propagation (BP) neural network was selected to predict cells density in this work. Figure 13 illustrates the concept of using BP neural network to predict the value of CDR. Besides the principal components extracted from FRF datasets, the value of index $A F D R$ of each sample provide an additional input to the neural network. As the index of $A F D R$ has been proved to be highly related to cells distribution in last section, it can help the neural network to achieve a fast convergence and good predictions. Among the 18 samples in the dataset, the first 14 samples are used for training neural network and the left 4 sample are used for validation.

As the number of samples are limited, it is more sensible to design and use a simple neural network rather than a complicated one. The BP neural network used here is designed to have only one hidden layer with few neurons. Several trials with different number of hidden layer neurons were carried out to test the differences on 


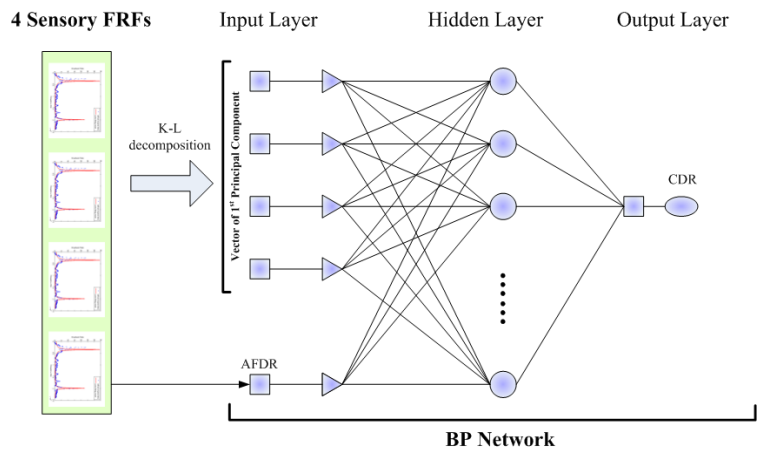

Figure 13: Schematic diagram of BP network used for cells identification

the normalized system error. It proves that the hidden layer with 5 neurons produces the best performance.

The training process of BP network herein establishes an approximate function (nonlinear regression) between the inputs and targets, through iteratively adjusting the weights and biases of network to meet a setting goal (mean square error). The training parameters can affect the network convergence speed as well as the final predication accuracy. Bad parameters may lead to very slow training processes or over-fitting results. Several tests were then carried out to find optimal training parameters. The final training parameters used in this work are selected as: moment rate is 0.9 , learning rate is 0.1 , the max error is 0.001 and the max number of iteration is 3000 .

\subsection{Prediction results}

Figure 14 demonstrates the prediction results of CDR on samples of No.15 No.18 from the trained BP network. The prediction results match very well with the CDR values calculated from corresponding LSM images, with erros within 10 percent.

\section{Conclusion and disscussion}

The experiments implemented in this research have examined the biosensing performance of a micromachined rectangular silicon membrane in a normal cell culture environment. The principals of biosensing used on the micro-membrane are based on the changes of its dynamic properties caused by cell adhesion. In contrast to previous research of biosensors, the bio-experiments on each type of micro-membranes were repeated many times. Initially, the shifts of resonant frequencies were employed to analyse the experimental results. The analytical results demonstrates that the rectangular micromembranes have capability on cell detection, under high-damping liquid conditions. Nevertheless, a fairly linear relationship of the micro-membrane sensitivity is rarely achieved. It reflects the complexity of rectangular micro-membrane in the applications of biosensing. Those results also reveal the issue of that a certain amount of difference of the biosensing sensitivity between two different micro-membranes exists, even they are of an identical type.

Further biosensing analysis of the micro-membrane is based on the novel methodology that uses an artificial neural network with a distributed sensing scheme to estimate the adhesive cell distribution. Karhunen-Loeve (K-L) decomposition method is successfully used to reduce the dimension of measured FRF datasets. A BP neural network is trained from a set of selected experimenal samples. The final predicted results on the other samples prove that this methodology can be successfully applied to identify the cell features. Significant advantages are discovered by applying this methodology in the biosensing analysis: (1)it is a robust algorithm and can repress the uncertainties in experimental measurements, comparing with using a single value as sensing parameter; (2)it is capble of eliminating the differences between diffferent substances of micro-membrane based biosensors; (3)it can overcome the inherent nonlinearity of sensing structure; (4)it is suitable to analyse the cells that are of very unregular shapes and nonuniform density, such as the EA.hy 926.

The work described in this paper is the first attempt of using the neural-network algorithm to perform the biosensing function of rectangular micro-membranes with a distributive sensory scheme. Much further research is required to develop more potential applications of this methodology in the field of biosensing. Although many repeated bio-experiments have been implemented in this work, the number of samples remains insufficient large for training a sophisticated neural network. Current predicted results of cell adhesion is only for the cell density spreading on the membrane sensing surface, which primarily reflects the weight information of cells. The distributive sensory data of membrane also provide the space information of the adhesive cells. Consequently, it is likely to predict the position, morphology and behaviours of living biological particles by using the proposed methodology. Such information are more useful in the biological applications than the weight information.

\section{ACKNOWLEDGMENT}

The authors would acknowledge the funding support from EPSRC committee. 


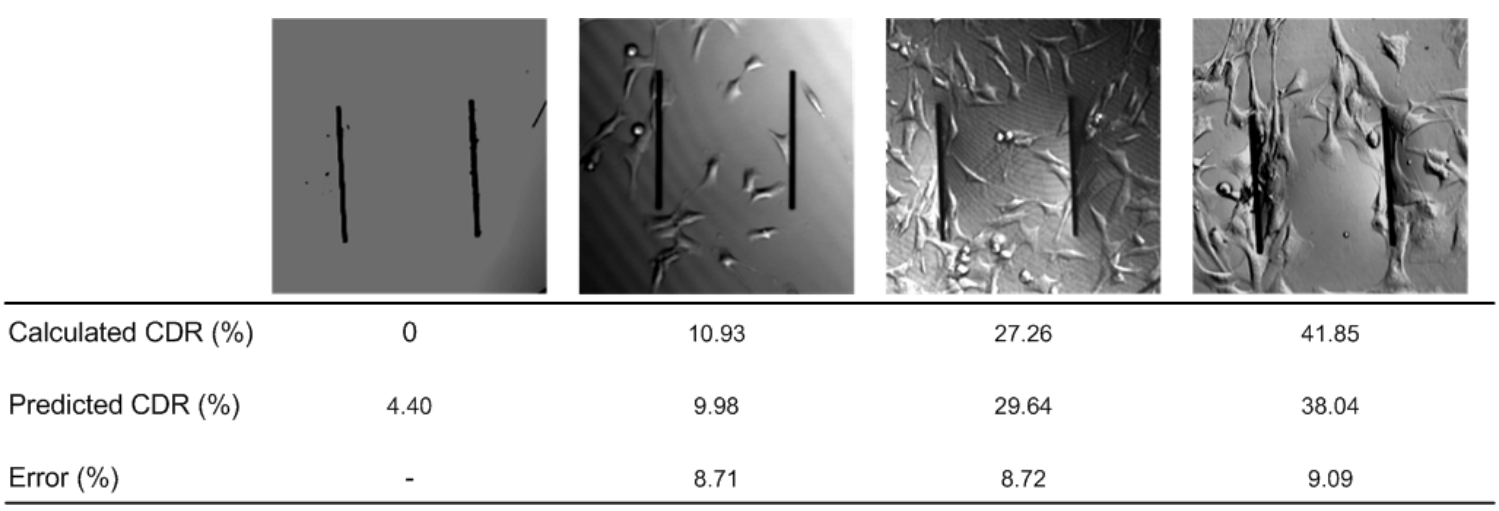

Figure 14: Predicted results on the CDR of No.15 No.18 samples using the trained BP neural network

[1] S. Mohanty, E. Kougianos, Biosensors: a tutorial review, IEEE Potentials 25 (2) (2006) 35-40.

[2] R. Raiteri, M. Grattarola, H.-J. Butt, P. Skladal, Micromechanical cantilever-based biosensors, Sensors and Actuators B 79 (23) (2001) 115-126.

[3] L. Carrascosa, M. Moreno, M. Alvarez, L. Lechuga, Nanomechanical biosensors: A new sensing tool, TrAC - Trends in Analytical Chemistry 25 (3).

[4] N. Lavrik, M. Sepaniak, P. Datskos, Cantilever transducers as a platform for chemical and biological sensors, Review of Scientific Instruments 75 (7) (2004) 2229-2253.

[5] K. W. Wee, G. Y. Kang, J. Park, J. Y. Kang, D. S. Yoon, J. H. Park, T. S. Kim, Novel electrical detection of label-free disease marker proteins using piezoresistive self-sensing microcantilevers, Biosensors and Bioelectronics 20 (10) (2005) 19321938.

[6] T. Xu, Z. Wang, J. Miao, L. Yu, C. Li, Micro-machined piezoelectric membrane-based immunosensor array, Biosensors and Bioelectronics 24 (4) (2008) 638-432.

[7] E. T. Carlen, M. S. Weinberg, C. E. Dub, A. M. Zapata, J. T. Borenstein, Micromachined silicon plates for sensing molecular interactions, Applied Physics Letters 89 (17) (2006) 173123173124.

[8] X. Ma, P. Brett, The performance of a 1-d distributive tactile sensing system for detecting the position, weight, and width of a contacting load, IEEE Transactions on Instrumentation and Measurement 51 (2) (2002) 331-6.

[9] B. M. Cowie, D. J. Webb, B. Tam, P. Slack, P. N. Brett, Distributive tactile sensing using fibre bragg grating sensors for biomedical applications, Proceedings of the First IEEE/RAS-EMBS International Conference on Biomedical Robotics and Biomechatronics, 2006, BioRob 20062006 (2006) 312-317.

[10] M. Elliott, X. Ma, P. Brett, Tracking the position of an unknown moving load along a plate using the distributive sensing method, Sensors and Actuators: A. Physical 138 (1) (2007) 28-36.

[11] Z. Chaudhry, A. Ganino, Damage detection using neural networks: an initial experimental study on debonded beams, Journal of Intelligent Material Systems and Structures 5 (4) (1994) 585-9.

[12] C. Zang, M. Imergun, Structural damage detection using artificial neural networks and measured frf data reduced via principal component prediction, Journal of Sound and Vibration 242 (5) (2001) 813-27.

[13] Y. Ni, X. Zhou, J. Ko, Experimental investigation of seismic damage identification using pca-compressed frequency response functions and neural networks, Journal of Sound and Vibration
290 (1-2) (2006) 242-63.

[14] J. Lee, S. Kim, Structural damage detection in the frequency domain using neural networks, Journal of Intelligent Material Systems and Structures 18 (8).

[15] R. Levin, N. Lieven, Dynamic finite element model updating using neural networks, Journal of Sound and Vibration 210 (5) (1998) 593-607.

[16] Z. Wu, X. Ma, P. Brett, J. Xu, Vibration analysis of submerged micro rectangular plates with distributed mass loading, Proceedings A of the Royal Society 465 (A) (2009) 205-216.

[17] B. Ilic, Y. Yang, H. Craighead, Virus detection using nanoelectromechanical devices, Applied Physics Letters 85 (13) (2004) 2604-2606.

[18] A. Gupta, D. Akin, R. Bashir, Single virus particle mass detection using microresonators with nanoscale thickness, Applied Physics Letters 84 (11) (2004) 1976-1978.

[19] X. Ma, A. Vakakis, L. Bergman, Karhunen-loeve analysis and order reduction of the transient dynamics of linear coupled oscillators with strongly nonlinear end attachments, Journal of Sound and Vibration 309 (3-5) (2008) 569-87. 